\title{
Complexity of syncope in elderly people: a comprehensive geriatric approach
}

\author{
CW Wong *
}

\begin{abstract}
A B S T R A C T
Syncope is a prevalent health problem among elderly people. It may be neurally mediated or caused by conditions such as orthostatic hypotension, postprandial hypotension, and cardiac disorders. A combination of different aetiologies is not uncommon in the elderly people. Many pathophysiological processes, including age-related physiological changes, co-morbidities, concomitant medication use, and prolonged bedrest, coexist and predispose elderly people to syncope; advanced age and cardiac syncope are associated with increased mortality. Recurrent syncope and its comorbidities, such as fall-related physical injury, negative psychological impact, and functional decline, may increase the dependency of elderly patients. Furthermore, the overlap between falls and syncope, and the interaction between syncope and
\end{abstract}

frailty complicate management. Available clinical guidelines for the management of syncope that focus on diagnosis, investigation, and treatment are therefore insufficient to address syncope in elderly patients. A comprehensive geriatric approach that considers an individual's functional and cognitive capacities, as well as medical and psychosocial aspects, would be more appropriate.

\section{Hong Kong Med J 2018;24:182-90}

DOI: 10.12809/hkmj176945

CW Wong *, FHKAM (Medicine), FHKCP

Department of Medicine and Geriatrics, Caritas Medical Centre, Shamshuipo, Kowloon, Hong Kong

* Corresponding author: chitwaiwong@hotmail.com

\section{Introduction}

Syncope is a transient loss of consciousness (LOC) and postural tone resulting from global cerebral hypoperfusion, followed by spontaneous and complete recovery and no neurological sequelae. This pathophysiology distinguishes syncope from other causes of transient LOC, including metabolic disorder, epileptic seizure, and transient ischaemic attack.

Syncope is prevalent in elderly populations. After a peak in younger populations (age, 10-30 years), the incidence of syncope increases sharply from 5.4 events per 1000 person-years in people aged 60 to 69 years to 11.1 events per 1000 personyears in those aged 70 to 79 years, and reaching 19.5 events per 1000 person-years in those aged 80 years or older. ${ }^{2}$ The incidence is similar for men and women but tends to increase in women of advanced age. The prevalence of syncope is high for the institutionalised elderly, at $23 \% .^{3}$ Syncope in elderly patients often presents atypically, such as with falls. Elderly patients may also have difficulty in recalling events. Therefore, the true incidence and prevalence of syncope are expected to be higher than those estimated in some previous studies.

Syncope is typically an isolated disease in young people. However, it is usually multifactorial and associated with many predisposing factors in the elderly people; thus, its management is challenging. In this article, the aetiology of syncope in the elderly people, pathophysiological factors that impair haemodynamic homeostasis, consequences of syncope, and relationships between syncope and falls and between syncope and frailty are discussed. Finally, a comprehensive approach for the management of syncope in elderly patients is recommended.

\section{Causes of syncope in the elderly people}

The causes of syncope are highly age-dependent. ${ }^{4}$ Reflex or neurally mediated syncope is the most common cause, particularly in younger patients. With increasing age, orthostatic hypotension $(\mathrm{OH})$ and cardiac syncope occur more frequently.

\section{Reflex or neurally mediated syncope}

Reflex syncope is a heterogeneous group of conditions, including vasovagal syncope, situational syncope, and carotid sinus syndrome, and is the most frequent cause of syncope in the elderly people $\left(44 \%\right.$ of cases). ${ }^{5}$ In reflex syncope, the cardiovascular reflexes that normally help control the circulation become intermittently inappropriate (eg, inappropriate vasodilation or bradycardia) in response to a trigger (eg, emotion or orthostatic stress).

Vasovagal syncope is the most common form 
of reflex syncope and is mediated by the vasovagal reflex. The most common triggers in elderly people are prolonged standing or sitting and use of vasodilator drugs. The classic prodromal features (pallor, diaphoresis, nausea, and warmth) are less prominent in elderly people.

Situational syncope occurs in conditions that trigger the Valsalva manoeuvre, such as urination, defaecation, coughing, and swallowing.

Syncope exacerbated by the carotid sinus reflex response is referred to as carotid sinus syndrome (CSS). It is related to underlying carotid sinus hypersensitivity, which is diagnosed when carotid sinus massage (CSM) produces asystole longer than 3 seconds (cardioinhibitory CSS) or a reduction in systolic blood pressure (SBP) by more than $50 \mathrm{~mm}$ $\mathrm{Hg}$ (vasodepressor CSS). Carotid sinus syndrome typically occurs in adults older than 50 years and is predominant in men. There is usually no identifiable trigger, but CSS may be precipitated by sudden head turning and wearing tight clothing around the neck. ${ }^{6}$ Carotid sinus syndrome is regarded as a significant cause of syncope and unexplained falls in the elderly people; as many as $45 \%$ of elderly patients presenting with syncope or unexplained falls demonstrate carotid sinus hypersensitivity. ${ }^{7,8}$

\section{Orthostatic hypotension}

Orthostatic hypotension $(\mathrm{OH})$ is prevalent in elderly people and in those who are frail, affecting up to $18 \%$ of people aged 65 years or older ${ }^{9}$ and up to $52 \%$ of institutionalised elderly patients. ${ }^{10}$ It also accounts for $30 \%$ of cases of syncope in patients aged 75 years or older. ${ }^{5}$ Orthostatic hypotension is defined as a sustained reduction in systolic blood pressure of

\section{老年人暈厥的複雜性：綜合性老年人評估方案} 王哲慧

暈厥是老年人普遍存在的健康問題。它可能是神經介導的或由起立性 低血壓、餐後低血壓和心藏疾病等情況引起。在老年人中, 有多於一 種不同病因並不罕見。許多病理生理過程容易導致老年人暈厥, 包括 與年齡相關的生理變化、合併症、多重用藥和長期臥床；而高齡和心 臟量厥則與死亡率增加有關。復發性暈厥及其合併症, 如與跌倒有關 的身體損傷、心理和功能衰退可能增加老年患者的依賴性。跌倒與量 硕的因果關係，以及暈厥與衰弱間的相互作用也為治療增添複雜性。 因此, 着重診斷、檢查和治療方面的量厥臨床治理指南並不足以解決 老年患者的暈厥問題, 而將個人醫療狀況, 身體機能, 認知能力及社 會心理因素納入全面評估的治療方案會更為合適。

at least $20 \mathrm{~mm} \mathrm{Hg}$ or in diastolic blood pressure of $10 \mathrm{~mm} \mathrm{Hg}$, within 3 minutes of standing. ${ }^{11}$ Because elderly patients may present beyond 3 minutes of standing and $\mathrm{OH}$ may not always be reproducible in elderly people, repeated measurement-preferably in the morning-may be required. Among factors that precipitate $\mathrm{OH}$ are age-related changes in the blood pressure regulatory mechanism, disease-related autonomic dysfunction, and numerous other factors that decrease cardiac output or total peripheral vascular resistance (Table). Occurrence of $\mathrm{OH}$ is significantly related to the number of co-morbidities and to potentially causative medications. ${ }^{12,13}$ However, supine systolic hypertension is often also present in elderly people with $\mathrm{OH}$ and further complicates management of $\mathrm{OH}$.

\section{Postprandial hypotension}

Postprandial hypotension $(\mathrm{PPH})$ is also common

TABLE. Aetiology and precipitating factors of syncope

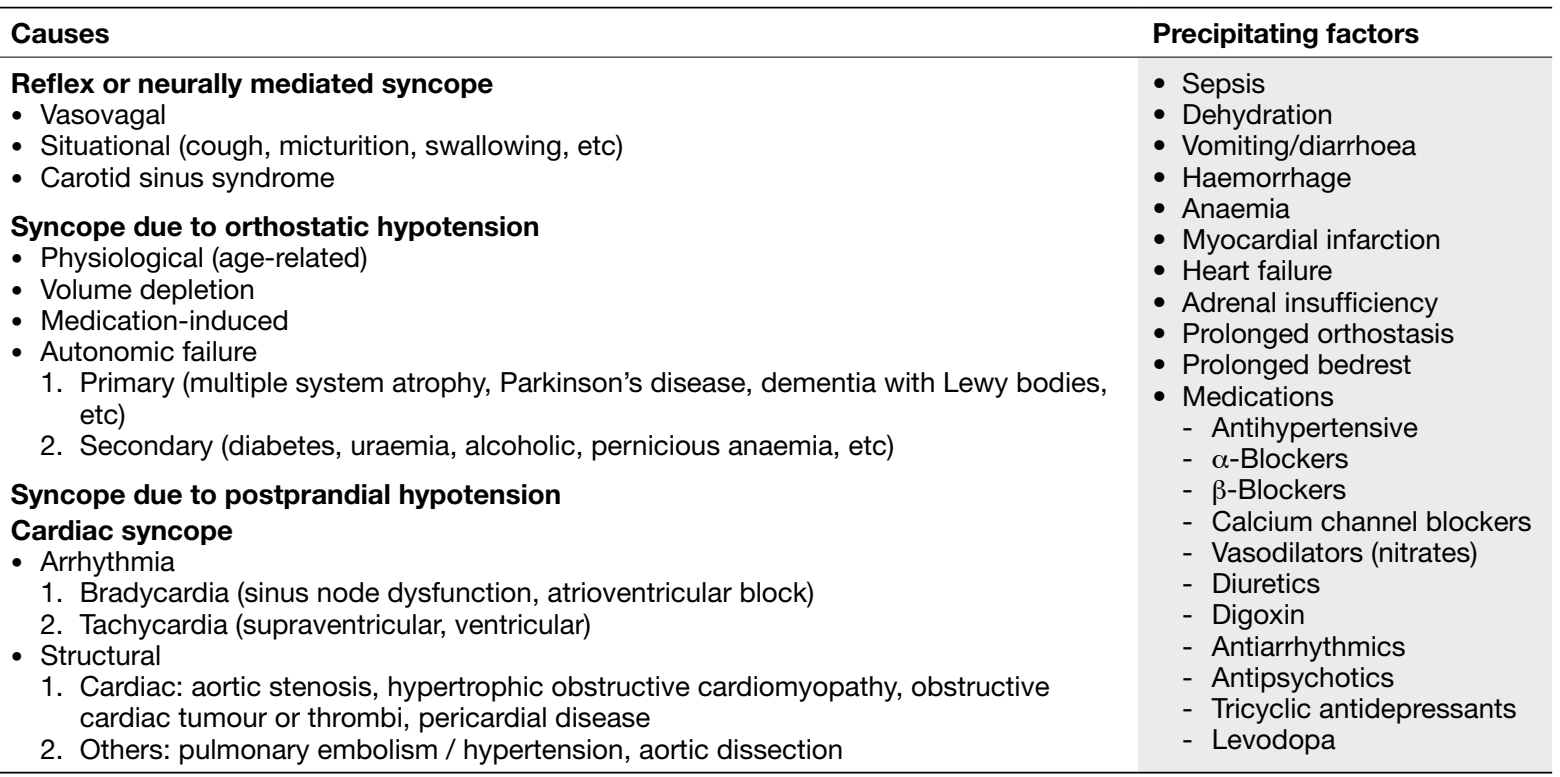


in the elderly people, with a prevalence reaching $59 \%$ in older Chinese men, especially in those older than 80 years. ${ }^{14}$ It is often under-recognised as a potential cause of syncope and is seldom mentioned in published clinical guidelines on syncope. In one study, PPH accounted for $8 \%$ of syncopal episodes. ${ }^{15}$ Postprandial hypotension is defined as a decline in systolic blood pressure of $20 \mathrm{~mm} \mathrm{Hg}$ or more, or to lower than $90 \mathrm{~mm} \mathrm{Hg}$, within 2 hours of starting a meal. ${ }^{16}$ Similar to OH, PPH is more likely to occur in the morning. Consuming a meal that is large or rich in carbohydrates also increases the risk of PPH. The condition is caused by the pooling of blood in the splanchnic vascular bed. Although PPH occurs frequently with $\mathrm{OH}$, the pathophysiological mechanisms of $\mathrm{OH}$ and $\mathrm{PPH}$ may be different. ${ }^{17}$ Postprandial hypotension is more likely to occur if patients have diabetes mellitus, hypertension, or Parkinson's disease, or if they take multiple concurrent medications, particularly diuretics. ${ }^{18,19}$

\section{Cardiac syncope}

Cardiac syncope accounts for up to $15 \%$ of cases of syncope in the elderly people. ${ }^{5,20}$ It is caused by impaired cardiac output due to arrhythmia or structural heart disease in which left ventricular blood flow is obstructed. Arrhythmia-either bradyarrhythmia or tachyarrhythmia-is the most common cause of cardiac syncope. Calcific degenerative aortic stenosis is the most common valvular lesion in the elderly people and also the most common structural cardiovascular cause of syncope..$^{21}$ In general, cardiac syncope is associated with a high mortality rate. ${ }^{2}$

\section{Unexplained syncope}

The prevalence of unexplained syncope has decreased as the use of diagnostic techniques, such as the tilt-table test and CSM, has increased. These techniques can identify reflex syncope and CSS in the elderly people. The reported proportion of elderly patients with unexplained syncope decreased from $40 \%$ in $1986^{22}$ to $10 \%$ in $2006 .{ }^{5}$ For elderly patients with syncope in whom the aetiology remains undetermined after extensive evaluation, it is important to look for a cardiac cause. In a study using an implantable loop recorder, arrhythmia accounted for $59 \%$ of all recurrences of syncope that would previously have been regarded as unexplained. ${ }^{23}$

\section{Multiple causes of syncope}

There are commonly multiple potential causes of syncope in elderly patients: in $23.5 \%$ of those aged 65 or older and in $13.4 \%$ of those younger than 65 years. ${ }^{24}$ Apart from older age, patients with atrial fibrillation or symptomatic heart failure, or those receiving drug therapy for cardiac conditions are more likely than others to have multiple causes of syncope. The presence of multiple causes also correlates with poor survival.

\section{Multiple risk factors in the elderly people}

Maintaining blood pressure at a constant level during a wide range of daily activities or conditions requires an intact cardiovascular system, baroreflex function, autonomic nervous system, and humoural regulatory mechanism. Age-related change in the blood pressure regulatory mechanism increases the susceptibility to syncope in elderly people. Baroreflex sensitivity is blunted by ageing, resulting in reduced heart rate and reduced vasoconstriction response to hypotensive stimuli. ${ }^{25}$ Furthermore, elderly people are prone to dehydration and reduced blood volume owing to reduced thirst sensation, reduction in renin-aldosterone activity, and elevation of atrial natriuretic peptide, which decreases the kidneys' capacity to conserve salt and water. ${ }^{26,27}$ The blunted baroreflex response and contracted blood volume, together with age-related diastolic dysfunction, can lead to low cardiac output and thus low cerebral blood flow. The latter predisposes elderly people to syncope under conditions causing hypotensive stress.

Co-morbidities and concomitant medication use can impair the adaptive response to hypotensive stress. Any conditions that contribute to haemodynamic stress or impair the blood pressure regulation mechanism, leading to decreased cardiac output or total peripheral vascular resistance (vasodilation), predispose the elderly people to reflex syncope and OH. Syncope can be induced by acute conditions, such as sepsis, myocardial infarction, cardiac arrhythmia, heart failure, haemorrhage, and dehydration. It can also be induced by chronic conditions such as chronic adrenal suppression from steroid use (which can produce hypovolaemia), as well as untreated or uncontrolled hypertension and coronary heart disease. Untreated or uncontrolled hypertension can increase the risk of $\mathrm{OH}$ by reducing baroreflex sensitivity and shifting the threshold for cerebral autoregulation to a higher blood pressure, ${ }^{28,29}$ whereas coronary heart disease is associated with carotid sinus hypersensitivity. ${ }^{30}$ In addition, patients presenting with $\mathrm{OH}$ may have autonomic insufficiency, which can be secondary to peripheral nervous system diseases, such as diabetes mellitus, alcoholism, chronic kidney disease, vitamin $B_{12}$ deficiency, and paraneoplastic disease. Autonomic insufficiency may also be due to central nervous system diseases, such as multiple system atrophy, dementia with Lewy bodies, and Parkinson's disease.

The Table lists the medications that may 
precipitate syncope. Although antihypertensivedrugs are commonly thought to increase the risk of postural blood pressure drop and thus $\mathrm{OH}$, clinical trials have shown inconsistent results. ${ }^{31}$ On the contrary, the reduction or normalisation of blood pressure by antihypertensive agents may even improve $\mathrm{OH}$ in elderly patients with hypertension. ${ }^{32}$ However, excessive blood pressure reduction and concurrent use of three or more antihypertensive drugs is independently associated with $\mathrm{OH} .{ }^{12,13}$ Diuretics, nitrates, antipsychotics, tricyclic antidepressants, and levodopa can also induce $\mathrm{OH}$. Drugs that can induce QT prolongation and torsades de pointes include antiarrhythmics and antipsychotics. ${ }^{33}$ Drugs that can cause bradyarrhythmia include amiodarone, beta-blockers, calcium channel blockers, and digoxin. Because of age-related changes in pharmacokinetics and pharmacodynamics, the adverse effects and interactions of these drugs are further exacerbated in the elderly people.

Bedrest is common in patients with acute illness or in those who are frail or chronically ill. After prolonged bedrest, however, elderly patients can develop syncope when sitting up. Prolonged bedrest can cause deconditioning in the musculoskeletal and cardiovascular system, as well as pressure natriuresis, which in turn induces blood volume contraction. These conditions can aggravate $\mathrm{OH}$, syncope, and fall. ${ }^{34}$

All of the above pathophysiological processes can act together to impair cardiovascular compensation for haemodynamic stress in elderly people and thereby complicate the management of syncope.

\section{Consequences of syncope}

Whether syncope independently increases the risk of overall or cardiac mortality remains controversial. Nonetheless, among patients with syncope, mortality generally tends to increase with advancing age and in those with cardiac syncope and $\mathrm{OH} .{ }^{35-39}$ A 2-year study found that the overall mortality rate was $0 \%$ in patients aged 65 to 69 years, increasing to $14 \%, 22 \%$, and $43 \%$ in patients aged 70 to 79 years, 80 to 89 years, and $\geq 90$ years, respectively. ${ }^{37}$ Syncope of cardiac cause was noted to have higher 5-year mortality (51\%) than non-cardiac (30\%) or unknowncause (24\%) mortality. ${ }^{38}$ Orthostatic hypotension is associated with a significantly increased risk of death for patients younger than 65 years (relative risk $=1.78 ; 95 \%$ confidence interval $=1.25-2.52$ ) but not for older patients. ${ }^{39}$ Overall prognosis is worse in patients with an underlying cardiac disease such as myocardial infarction, arrhythmia, structural cardiac defect, cardiomyopathy, or congestive heart failure, regardless of the occurrence or aetiology of syncope. ${ }^{36,40,41}$ The presence of multiple potential causes of syncope also predicts a lower survival rate as compared with a single cause of syncope (73\% vs $89 \%$ at 4 years). ${ }^{24}$ In contrast, patients with non-cardiac co-morbidities or no underlying heart disease are at low risk.

Patients with a history of syncope have an increased risk of recurrence. Patients with syncope have a $20 \%$ recurrence rate at 1 year, compared with a $2 \%$ rate of syncope in patients without prior syncope. ${ }^{40}$ The recurrence rate is even higher in the institutionalised elderly, at $30 \% .^{3}$ Syncope recurs more frequently in older patients at 2 years, with a rate of $28 \%$ in patients aged 65 to 79 years, $37 \%$ in those aged 80 to 89 years, and $43 \%$ in those older than 90 years. ${ }^{37}$ Other factors predicting recurrence include the concurrent use of multiple medications with known adverse effects of $\mathrm{OH}$ or syncope and cardiac co-morbidities, particularly atrial fibrillation, atrioventricular or left bundle branch block, or aortic valve stenosis. ${ }^{42}$ Recurrence may not be related to the aetiology of syncope, although a higher recurrence in patients affected by reflex and unexplained syncope has been reported. ${ }^{36}$

Besides physical injuries and disabilities due to syncope-related falls, depression and fear of falling after syncope may reduce elderly patients' functional capacity and mobility, and can result in institutionalisation. Furthermore, syncope can negatively affect quality of life, ${ }^{43}$ particularly in patients who are older, have multiple co-morbidities, and experience recurrent episodes of syncope. ${ }^{44}$

\section{Syncope and falls}

There is an overlap between syncope and falls in the elderly people; syncope causes falls and some falls are due to syncope. Although taking an accurate history of an event allows the differentiation between syncope and fall, the history is often unreliable in the elderly people because of poor recall and lack of witnesses. Many elderly patients will only recall the fall but not realise they fainted; amnesia is common (up to $42 \%$ ) for witnessed syncope, even in cognitively normal elderly patients. ${ }^{6-8,45}$ This is particularly the case for CSS: in one study, $21 \%$ of patients with CSS presented with falls alone and $27 \%$ failed to recall the syncope event. ${ }^{6}$ Furthermore, frequently reported reasons for falls in the elderly people, such as muscle weakness, arthritis, gait and balance problems, visual impairment, functional decline, depression, cognitive impairment, polypharmacy, and environmental factors, ${ }^{46,47}$ may distract clinicians from searching for syncope.

Nonetheless, multiple co-morbidities and polypharmacy associated with advancing age predispose elderly people to both falls and syncope. Moderatehaemodynamic changes thatareinsufficient to cause syncope may cause falls in elderly patients with gait balance instability or slow protective reflexes. ${ }^{48}$ Such falls may be indistinguishable from 
syncope in elderly patients. Considering that syncope may be a component of fall in elderly patients, especially in those with unwitnessed or recurrent unexplained fall, assessment of cardiovascular status and postural blood pressure, as well as further investigation for accurate diagnosis and treatment, are recommended. ${ }^{47}$

\section{Syncope and frailty}

Frailty that leads to diminished functional reserve and adverse health outcomes is defined as the presence of three of more of the following criteria: unintentional weight loss (10 lbs $[\sim 4.5 \mathrm{~kg}]$ in the past year), selfreported exhaustion, weakness (grip strength), slow walking speed, and low physical activity. ${ }^{49}$ The relationship between frailty and syncope is not well described in the literature. However, there is an interaction between ageing, co-morbidities, and the development of frailty (Fig 1). Co-morbidities are common among the elderly people. As many as $82 \%$ of people aged 65 years or older have one or more chronic conditions. ${ }^{50}$ Advancing age and comorbidities, in turn, increase the likelihood of being frail. Conversely, frail people are likely to have more co-morbidities. ${ }^{51}$ Age-related physiological changes and co-morbidities also increase the risk of syncope and falls. Thus, an episode of syncope with a fall may act as an acute stressor in the elderly people. The decreased physiological reserve in frail elderly patients inhibits their response to acute stressors. This inhibited response leads to rapid functional decline and results in hospitalisation, dependency, and institutionalisation (Fig 1).

\section{Comprehensive geriatric approach}

According to clinical guidelines, ${ }^{1,52}$ initial evaluation for syncope starts with careful recording of the patient's history, physical examination (including orthostatic blood pressure measurement), and

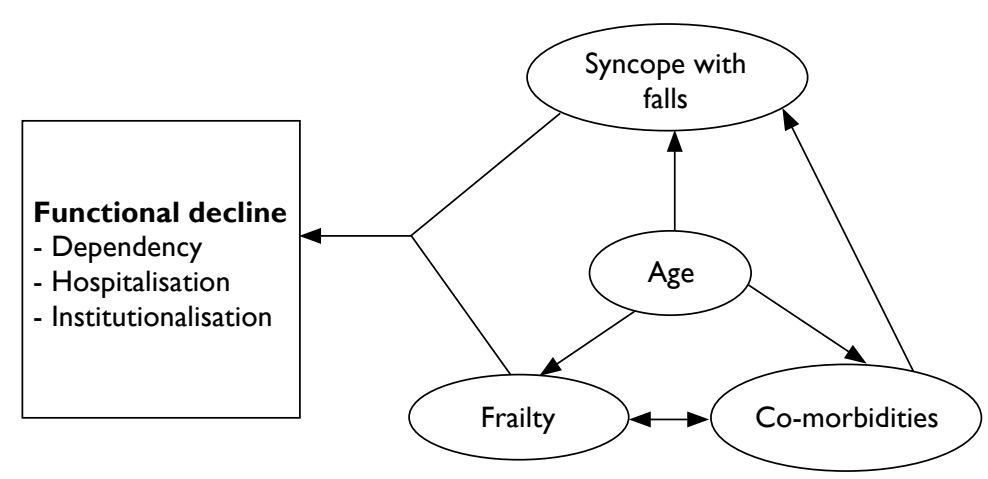

FIG I. Interaction between syncope, age, frailty, and co-morbidities electrocardiography (ECG). On the basis of these findings, additional examinations such as Holter monitoring, CSM, tilt-table test, echocardiography, and blood tests may be performed. The purpose of the initial evaluation is to differentiate syncopal from non-syncopal conditions, to determine the cause of syncope, and to stratify the risk of major cardiovascular events or death. Predictors of high cardiovascular risk are the presence of structural heart disease or coronary artery disease (eg, heart failure, low left ventricular ejection fraction, and previous myocardial infarction), palpitation before syncope, syncope during exertion or in the supine position, and ECG features suggesting arrhythmic disturbance. Initial evaluations can identify a certain or highly likely cause of syncope in $63 \%$ of patients. ${ }^{53}$ If the cause remains uncertain, further investigation is indicated. The subsequent tests are based on the findings of the initial evaluation and suspected aetiology: tilt-table test for reflex syncope or $\mathrm{OH}$; CSM for elderly people with unexplained falls; Holter monitoring or external/ implantable loop recording to detect arrhythmia in recurrent unpredictable syncope; echocardiography for structural heart disease and cardiac function, especially in the presence of abnormal findings of ECG or cardiovascular examination or suspicion from history; or neurological evaluation for autonomic failure or neurological conditions that are difficult to differentiate from syncope. Treatment depends on the underlying causes and usually includes the following:

- Patient education to avoid triggers (prolonged standing or extreme environments), to recognise prodromal symptoms, and to perform manoeuvres to abort episodes of syncope

- Review and adjustment of the patient's medication

- Non-pharmacotherapies for $\mathrm{OH}$, including increasing fluid and salt intake, eating frequent small meals and low-carbohydrate meals, raising the head of the bed when sleeping, slowly rising from the supine position, wearing compression stockings or an abdominal binder (compression belt), and exercising

- Pharmacotherapies for $\mathrm{OH}$, including fludrocortisone, midodrine, and octreotide

- Cardiac interventions for cardiac syncope or CSS with cardioinhibitory response, including cardiac pacing or an implantable cardiac defibrillator

However, as outlined in this article, syncope in elderly people has certain complexities-namely, (1) there is a marked overlap between syncope, fall, and dizzy spell, and atypical presentations make diagnosis difficult; (2) the syncope event is often multifactorial, with many predisposing factors; and (3) management is often complicated by underlying co-morbidities, concomitant medication 
use, cognitive and functional decline, and declined psychosocial support. Therefore, the above standard structural approach is insufficient to address syncope in elderly patients. Comprehensive geriatric assessment, "a multidimensional interdisciplinary diagnostic process focused on determining a frail elderly person's medical, psychological and functional capacity in order to develop a coordinated and integrated plan for treatment and long term follow up", is recommended. ${ }^{54}$ This comprehensive approach is both a diagnostic and a therapeutic process and involves a multidisciplinary team (physician, nurse, physiotherapist, occupational therapist, and social worker) with the collective goal of improving care outcomes and quality of life for elderly people. When an elderly patient is referred for syncope, an unexplained fall, or dizziness, comprehensive geriatric assessment is applied to obtain a thorough history and physical examination, together with assessments for co-morbidities, frailty, cognitive and functional status, psychological and social variables, and medication review (Fig 2). After extensive evaluation, a problem list is generated with details of the identified syncope event and its cause or predisposing factors, whether the patient is at high risk of cardiovascular event or death, and any cognitive or functional impairments or psychosocial problems. Then, multidisciplinary interventions are integrated with the patient's or caregiver's preferences, so as to conserve the patient's health status, improve psychosocial support, and maintain the patient's independence and community living situation. The following should be considered in the management of syncope:

- Because of the overlap between syncope and falls, comprehensive geriatric assessment for syncope should be applied to include elderly people with falls, especially unexplained falls.

- Although the tilt-table test and CSM are regarded as safe in elderly people, the patient's tolerance to such tests should be considered, especially in frail patients.

- The extent of investigations depends on the patient's risk of cardiovascular events or death, and the severity of the symptoms and the frequency of recurrence. For example, for patients with a single episode who are at low risk of cardiac syncope, a test to confirm reflex syncope may be unnecessary.

- Stepped management starts with nonpharmacotherapy (such as education, medication adjustment, and physical manoeuvres), then pharmacotherapy if the patient's condition does not improve.

An example of comprehensive geriatric assessment for elderly people referred for syncope is presented in the following case scenario. A 75-yearold woman was admitted to hospital after an episode of transient LOC. She reported walking down the street then feeling dizzy, passing out, and falling on the ground. There were no eyewitnesses. The patient recovered spontaneously but could not recall the duration of LOC. She denied any preceding events such as palpitation or chest discomfort, except dizziness. The patient reported having experienced similar episodes twice in the previous 2 years, once while standing and once while walking. Her medical history included hypertension, type 2 diabetes mellitus, ischaemic heart disease, and knee osteoarthritis, for which she was taking ramipril, metformin, metoprolol, amlodipine, isosorbide mononitrate, and amitriptyline. The patient lived alone, walked unaided, and went out shopping daily. She expressed concerned about recurrent dizziness and falls, which had restrained her social activities. Physical examination revealed postural blood pressure drop from $110 / 60$ to $90 / 50 \mathrm{~mm} \mathrm{Hg}$ within 3 minutes of standing, normal heart sound, varus deformities in both knees due to osteoarthritis, and lower-limb weakness with muscle power grade 4/5.

\section{Evaluation}

- History

- Physical examination, including orthostatic blood pressure, cardiovascular, and neurological examinations

- Electrocardiography

- Further investigations for syncope as needed

- Co-morbidity assessment

- Medication review

- Medication compliance

- Mobility, gait and balance, muscle strength, and fall risk assessment

- Cognitive assessment

- Functional assessment (basic and extended activities of daily living)

- Frailty assessment

- Psychosocial assessment

- Nutritional assessment

\section{Interventions}

- Specific treatment for underlying cause, including patient's preference

- Non-pharmacological treatment (elevation of head of bed, compression stockings, abdominal binder [compression belt])

- Minimisation or modification of drug regimen

- Patient and caregiver education to avoid syncope (maintain hydration; avoid prolonged orthostatic position, heat and crowd places, and getting up quickly)

- Suitable walking aids

- Rehabilitation; gait and balance training

- Environmental modification or adaptation

- Psychosocial support

FIG 2. Comprehensive geriatric assessment for syncope in elderly patients 
Laboratory studies revealed a haemoglobin level of $100 \mathrm{~g} / \mathrm{L}$ with a mean corpuscular volume of 98 $\mathrm{fL}$; serum vitamin $\mathrm{B}_{12}, 120 \mathrm{pmol} / \mathrm{L}$; random serum glucose, $6 \mathrm{mmol} / \mathrm{L}$; and haemoglobin $\mathrm{A}_{1 \mathrm{c}}$, 5.8\%. Results of renal and liver function tests and ECG were normal. A nurse, a physiotherapist, and an occupational therapist also assessed the patient.

Relevant problems included the following:

- Causes and predisposing factors for syncope: $\mathrm{OH}$, vitamin $\mathrm{B}_{12}$ deficiency (probably related to long-term metformin intake), antihypertensive agent use, and amitriptyline use

- Fair drug compliance: sometimes forgot to take drugs or increased amitriptyline dosage because of pain

- Good diabetic control with haemoglobin $\mathrm{A}_{1 \mathrm{c}}$ of $5.8 \%$; possible risk of hypoglycaemia

- Varus deformity due to osteoarthritis of the knee; lower limb weakness, with risk of falls

- Living environment: no lift; needed to climb 2 flights of stairs

- Anxiety about recurrent dizziness and falls; poor psychosocial support because there were no close relatives in Hong Kong

Interventions implemented in this case were as follows:

- Physician: discontinued metformin therapy and arranged patient blood glucose monitoring, decreased amlodipine dosage and arranged blood pressure monitoring, initiated vitamin $\mathrm{B}_{12}$ replacement, discontinued amitriptyline, added paracetamol for pain relief with dosage titration and monitored response, and arranged regular follow-up

- Community nurse service: arranged home visits to monitor blood pressure and blood glucose, provide education on manoeuvres to minimise postural dizziness, and administer and supervise drugs intake

- Physiotherapist and occupational therapist: referred the patient to geriatric day hospital for muscle strengthening and mobility and activities of daily life training, and prescribed walking aids

- Social worker: arranged home support services such as meal delivery, household cleaning, and personal assistant service.

\section{Conclusions}

In younger patients, vasovagal syncope is the predominant aetiology of syncope. In contrast, CSS, OH, PPH, and cardiac syncope become more prevalent with advancing age. Multiple aetiologies are frequently observed in elderly patients. Interactions among age-related physiological changes, comorbidities, and concomitant medication use impair haemodynamic homeostasis and predispose elderly people to syncope. These factors, together with cognitive impairment, functional decline, and frailty associated with advanced age, make the management of syncope in elderly patients complex. Structured clinical guidelines are insufficient to manage syncope in elderly patients. Use of a comprehensive geriatric approach to integrate an individual's co-morbidities, cognitive and functional capacities, and medical and psychosocial aspects is more appropriate and beneficial for elderly patients so as to maintain functional state and quality of life.

\section{Declaration}

The author has no conflicts of interest to disclose.

\section{References}

1. Task Force for the Diagnosis and Management of Syncope; European Society of Cardiology (ESC); European Heart Rhythm Association (EHRA); Heart Failure Association (HFA); Heart Rhythm Society (HRS), Moya A, Sutton $\mathrm{R}$, Ammirati F, et al. Guidelines for the diagnosis and management of syncope (version 2009). Eur Heart J 2009;30:2631-71.

2. Soteriades ES, Evans JC, Larson MG, et al. Incidence and prognosis of syncope. N Engl J Med 2002;347:878-85.

3. Lipsitz LA, Wei JY, Rowe JW. Syncope in an elderly, institutionalised population: prevalence, incidence, and associated risk. Q J Med 1985;55:45-54.

4. Strickberger SA, Benson DW, Blaggioni L, et al. AHA/ ACCF scientific statement on the evaluation of syncope: from the American Heart Association Councils on Clinical Cardiology, Cardiovascular Nursing, Cardiovascular Disease in the Young, and Stroke, and the Quality of Care and Outcomes Research Interdisciplinary Working Group; and the American College of Cardiology Foundation in Collaboration with the Heart Rhythm Society. J Am Coll Cardiol 2006;47:473-84.

5. Ungar A, Mussi C, Del Rosso A, et al. Diagnosis and characteristics of syncope in older patients referred to geriatric departments. J Am Geriatr Soc 2006;54:1531-6.

6. Kenny RA, Traynor G. Carotid sinus syndrome-clinical characteristics in elderly patients. Age Ageing 1991;20:44954.

7. McIntosh SJ, Lawson J, Kenny RA. Clinical characteristics of vasodepressor, cardioinhibitory, and mixed carotid sinus syndrome in the elderly. Am J Med 1993;95:203-8.

8. Davies AJ, Steen N, Kenny RA. Carotid sinus hypersensitivity is common in older patients presenting to an accident and emergency department with unexplained fall. Age Ageing 2001;30:289-93.

9. Rutan GH, Hermanson B, Bild DE, Kittner SJ, LaBaw F, Tell GS. Orthostatic hypotension in older adults. The Cardiovascular Health Study. CHS Collaborative Research Group. Hypertension 1992;19(6 Pt 1):508-19.

10. Ooi WL, Barrett S, Hossain M, Kelley-Gagnon M, Lipsitz LA. Patterns of orthostatic blood pressure change and their clinical correlates in a frail, elderly population. JAMA 1997;277:1299-304.

11. Freeman R, Wieling W, Axelrod FB, et al. Consensus statement on the definition of orthostatic hypotension, neutrally mediated syncope and the postural tachycardia syndrome. Auton Neurosci 2011;161:46-8.

12. Kamaruzzaman S, Watt H, Carson C, Ebrahim S. The association between orthostatic hypotension and 
medication use in British women's heart and health study. Age Ageing 2010;39:51-6.

13. Poon IO, Braun U. High prevalence of orthostatic hypotension and its correlation with potentially causative medications among elderly veterans. J Clin Pharm Ther 2005;30:173-8.

14. Zou X, Cao J, Li JH, et al. Prevalence of and risk factors for postprandial hypotension in older Chinese men. J Geriatr Cardiol 2015;12:600-4.

15. Lipsitz LA, Pluchino FC, Wie JY, Rowe JW. Syncope in institutionalized elderly: the impact of multiple pathological conditions and situational stress. J Chronic Dis 1986;39:619-30.

16. Jansen RW, Lipsitz LA. Postprandial hypotension: epidemiology, pathophysiology, and clinical management. Ann Intern Med 1995;122:286-95.

17. Vloet LC, Pel-Little RE, Jansen PA, Jansen RW. High prevalence of postprandial and orthostatic hypotension among geriatric patients admitted to Dutch hospitals. J Gerontol A Biol Sci Med Sci 2005;60:1271-7.

18. Puisieux F, Bulckaen H, Fauchais AL, Drumez S, SalomezGranier F, Dewailly P. Ambulatory blood pressure monitoring and postprandial hypotension in elderly persons with falls or syncopes. J Gerontol A Biol Sci Med Sci 2000;55:M535-40.

19. Luciano GL, Brennan MJ, Rothberg MB. Postprandial hypotension. Am J Med 2010;123:281.e1-6.

20. Galizia G, Abete P, Mussi C, et al. Role of early symptoms in assessment of syncope in elderly people: results from the Italian group for the study of syncope in the elderly. J Am Geraitr Soc 2009;57:18-23.

21. Iivanainen AM, Lindroos M, Tilvis R, Heikklä J, Kupari M. Natural history of aortic valve stenosis of varying severity in the elderly. Am J Cardiol 1996;78:97-101.

22. Kapoor W, Snustad D, Peterson J, Wieand HS, Cha R, Karpf M. Syncope in the elderly. Am J Med 1986;80:41928.

23. Edvardsson N, Frykman V, van Mechelen R, et al. Use of an implantable loop recorder to increase the diagnostic yield in unexplained syncope: results from the PICTURE registry. Europace 2011;13:262-9.

24. Chen LY, Gersh BJ, Hodge DO, Wieling W, Hammill SC, Shen WK. Prevalence and clinical outcomes of patients with multiple potential causes of syncope. Mayo Clin Proc 2003;78:414-20.

25. Lipsitz LA. Altered blood pressure homeostasis in advancing age: clinical and research implications. J Gerontol 1989;44:M179-83.

26. Bauer JH. Age-related changes in the renin-aldosterone system, Physiological effect and clinical implication. Drugs Aging 1993;3:238-45.

27. Davis KM, Fush LC, Minaker KL, Elahi D. Atrial natriuretic peptide levels in the elderly: differentiating normal aging changes from disease. J Gerontol A Biol Sci Med Sci 1996;51:M95-101.

28. Bristow JD, Honour AJ, Pickering GW, Sleight P, Smith HS. Diminished baroreflex sensitivity in high blood pressure. Circulation 1969;39:48-54.

29. Strandgaard S. Autoregulation of cerebral blood flow in hypertensive patients. The modifying influence of prolonged antihypertensive treatment on the tolerance to acute, drug-induced hypotension. Circulation 1976;53:7207.
30. Brown KA, Maloney JD, Smith $\mathrm{CH}$, Haritzler GO, Ilstrup DM. Carotid sinus reflex in patients undergoing coronary angiography: relationship of degree and location of coronary artery disease to response to carotid sinus massage. Circulation 1980;62:697-703.

31. Hajjar I. Postural blood pressure changes and orthostatic hypotension in the elderly patient: impact of antihypertensive medications. Drugs Aging 2005;22:55-68.

32. Masuo K, Mikami H, Ogihara T, Tuck ML. Changes in frequency of orthostatic hypotension in elderly hypertensive patients under medications. Am J Hypertens 1996;9:263-8.

33. Yap YG, Camm AJ. Drug induced QT prolongation and torsades de pointes. Heart 2003;89:1363-72.

34. Stuempfle KJ, Drury DG. The physiological consequences of bed rest. J Exerc Physiol Online 2007;10:32-41.

35. Alshekhlee A, Shen WK, Mackall J, Chelimsky TC. Incidence and mortality rates of syncope in the United States. Am J Med 2009;122:181-8.

36. Racco F, Sconocchini C, Alesi C, Zappelli L, Pratillo G. Long-term follow-up after syncope. A group of 183 patients observed for 5 years [in English, Italian]. Minerva Cardioangiol 2000;48:69-78.

37. Ungar A, Galizia G, Morrione A, et al. Two-year morbidity and mortality in elderly patients with syncope. Age Ageing 2011;40:696-702.

38. Kapoor WN. Evaluation and outcome of patients with syncope. Medicine (Baltimore) 1990;69:160-75.

39. Ricci F, Fedorowski A, Radico F, et al. Cardiovascular morbidity and mortality related to orthostatic hypotension: a meta-analysis of prospective observational studies. Eur Heart J 2015;36:1609-17.

40. Kapoor WN, Hanusa BH. Is syncope a risk factor for poor outcomes? Comparison of patients with and without syncope. Am J Med 1996;100:646-55.

41. Middlekauff HR, Stevenson WG, Stevenson LW, Saxon LA. Syncope in advanced heart failure: high risk of sudden death regardless of origin of syncope. J Am Coll Cardiol 1993;21:110-6.

42. Ruwald MH, Hansen ML, Lamberts M, et al. Comparison of incidence, predictors, and the impact of co-morbidity and polypharmacy on the risk of recurrent syncope in patients $<85$ years versus $\geq 85$ years of age. Am J Cardiol 2013;112:1610-5.

43. Kenny RA, Bhangu J, King-Kallimanis BL. Epidemiology of syncope/collapse in younger and older Western patient populations. Prog Cardiovasc Dis 2013;55:357-63.

44. van Dijk N, Sprangers MA, Boer KR, Colman N, Wieling W, Linzer M. Quality of life within one year following presentation after transient loss of consciousness. Am J Cardiol 2007;100:672-6.

45. O’Dwyer C, Bennett K, Langan Y, Fan CW, Kenny RA. Amnesia for loss of consciousness is common in vasovagal syncope. Europace 2011;13:1040-5.

46. Rubenstein LZ, Josephson KR. The epidemiology of falls and syncope. Clin Geriatr Med 2002;18:141-58.

47. Guideline for the prevention of falls in older persons. American Geriatric Society, British Geriatrics Society, and American Academy of Orthopaedic Surgeons Panel on Falls Prevention. J Am Geriatr Soc 2001;49:664-72.

48. Kenny RA, Parry SW. Syncope-related falls in the elderly. J Geriatr Cardiol 2005;2:74-83.

49. Fried LP, Tang CM, Walston J, et al. Frailty in older adults: 
evidence for a phenotype. J Gerontol A Biol Sci Med Sci 2001;56:M146-56.

50. Wolff JL, Starfield B, Anderson G. Prevalence, expenditures, and complication of multiple chronic conditions in the elderly. Arch Intern Med 2002;162:2269-76.

51. Wong $\mathrm{CH}$, Weiss $\mathrm{D}$, Sourial $\mathrm{N}$, et al. Frailty and its association with disability and comorbidity in a community-dwelling sample of seniors in Montreal: a cross-sectional study. Aging Clin Exp Res 2010;22:54-62.

52. Shen WK, Sheldon RS, Benditt DG, et al. 2017 ACC/ AHA/HRS guideline for the evaluation and management of patients with syncope: a report of the American College of Cardiology/American Heart Association Task Force on Clinical Practice Guidelines and the Heart Rhythm Society. J Am Coll Cardiol 2017;70:e39-110.

53. van Dijk N, Boer KR, Colman N, et al. High diagnostic yield and accuracy of history, physical examination, and ECG in patients with transient loss of consciousness in FAST: the Fainting Assessment study. J Cardiovasc Electophysiol 2008;19:48-55.

54. Rubenstein LZ, Struck AE, Siu AL, Wieland D. Impacts of geriatric evaluation and management programs on defined outcomes: overview of the evidence. J Am Geriatr Soc 1991;39(9 Pt 2):8S-16S. 